Revista de Ciencias Sociales - Número 64 (2014) - Páginas 67-98

El Código Civil y las contradicciones de nuestra historia del derecho

\title{
EL CÓDIGO CIVIL Y LAS CONTRADICCIONES DE NUESTRA HISTORIA DEL DERECHO
}

\author{
THE CIVIL CODE AND THE CONTRADICTIONS \\ OF OUR HISTORY OF LAW
}

\author{
JAVIER VALLE SILVA* \\ Universidad Viña del Mar \\ jvalle@uvm.cl
}

\section{Resumen}

Nuestra cultura jurídica está vertebrada por el Código Civil, a partir de él hemos estudiado, enseñado e imaginado todo nuestro Derecho. Por ello, en este artículo se pretende exponer la visión que un influyente y prestigiado colectivo de historiadores del derecho tiene del origen y significado del Código Civil. Ellos lo ubican en una continuidad sustantiva con el derecho indiano. A partir de esta idea es posible encontrar tres contradicciones muy relevantes entre el trabajo historiográfico de la Escuela Chilena de Historia del Derecho y las ideas que implícita (y a veces explícitamente) defienden.

\section{Palabras claves} jurídica.

Historiografía jurídica, historia del derecho, Código Civil, cultura

\footnotetext{
* Doctorando en Derecho, Universidad de Chile. Artículo recibido el 12 de diciembre de 2013 y aceptado el 10 de marzo de 2014.
}

Revista de Ciencias Sociales - Número 64 (2014) - Universidad de Valparáíso - ISSN 0716-7725-Valparaíso, Chile 


\begin{abstract}
Our legal culture is structured by the Civil Code, from whom we have studied, taught and imagined all our law. Therefore, this article aims to present the vision that an influential and prestigious group of legal historians have about the origin and meaning of the Civil Code. They place it in a substantive continuity with the Law of the Indies. From this idea is possible to find three very important contradictions between the historiographical work of the Chilean School of Legal History and the ideas implicit (and sometimes explicit) that they defend.
\end{abstract}

\title{
Keywords
}

Legal historiography, legal history, Civil Code, legal culture.

\section{Explicación preliminar}

Si hay algo que no se ha hecho en nuestra academia es reflexionar sobre su propia historia intelectual ${ }^{1}$ y cómo ésta determina o influye en nuestra cultura jurídica. Es necesario hacerlo porque creo que es difícil no compartir la idea de que el Derecho no está constituido sólo por normas, sino que también lo está por ideas, prejuicios y valores (que lo definen de modo tan determinante como las normas) y que se transmiten desde la cátedra, a través de los manuales o por medio de otras prácticas más informales en la enseñanza del derecho. En este trabajo buscaré hacer algo así. Me he concentrado en la historia del derecho, particularmente en una orientación o corriente historiográfica muy

1. Hay por supuesto excepciones en los que se acomete esta tarea o se hace de modo tangencial, los ejemplos que he consultado son: MATUS ACUÑA, Jean Pierre: "El positivismo en el derecho penal chileno: análisis sincrónico y diacrónico de una doctrina de principios del siglo XX que se mantiene vigente". Revista de derecho (Valdivia), 2007, vol.20, n.1, pp. 175-203.; MATUS ACUÑA, Jean Pierre: "Origen, consolidación y vigencia de la Nueva Dogmática Chilena (ca. 1955 1970)”. Política criminal, 2011, vol.6, n.11, pp. 44-105; FERRADA BÓRQUEZ, Juan Carlos: "El sistema de derecho administrativo chileno: una revisión crítica desde una perspectiva histórica”. En XXXV Jornadas Chilenas de Derecho Público, Edeval, Valparaíso, 2006, pp. 207-231.

Facultad de Derecho y Ciencias Sociales - Universidad de Valparaíso - Chile 
influyente. ¿Y por qué la historia del derecho?; la razón estriba en que resulta bastante obvio que los operadores jurídicos se informan de lo que nuestro derecho fue por medio de las clases y publicaciones de los historiadores del Derecho y normalmente los juicios que se van a formar de los ordenamientos anteriores, sus modificaciones y las ideas que las inspiraron están determinadas por esas narraciones. La historia del derecho crea (aunque sea implícita o silenciosamente) un conjunto de ideas acerca del fenómeno jurídico y ayuda (o debería ayudar) a generar una capacidad para imaginar órdenes alternativos a partir de experiencias anteriores. En cuanto a las razones para preferir una corriente en particular, básicamente es por su relevancia, porque sus integrantes han divulgado sus ideas por décadas en algunas de las mejores facultades de derecho de Chile y en los primeros años de formación de muchos destacados abogados y profesores de derecho. Asimismo, en muchos lugares los profesores no necesariamente se sienten interpretados por sus ideas, pero ocupan sus manuales y sus programas para estructurar su docencia. Y generalmente sus obras son la fuente que la mayoría de los abogados y juristas ocupan para informarse acerca de nuestro pasado jurídico. Para terminar esta introducción, y para especificar aun más el propósito de este trabajo me interesa saber qué han dicho sobre nuestro Código Civil, por el impacto que tuvo de modo transversal en el sistema de fuentes y en el derecho chilenos.

\section{Nuestra Historia del derecho}

Existe en Chile una tradición de historiadores del derecho, integrada por Alamiro de Ávila, Manuel Salvat, Sergio Martínez, Bernardino Bravo Lira, Antonio Dougnac y Javier Barrientos². Ellos constituyen algo así como la imagen que tenemos de quiénes son y qué hacen los historiadores del derecho. Cabe mencionar que no obstante no son los únicos que han cultivado la historia del derecho en Chile, si

2. Tal vez he omitido a algún otro profesor, pero creo no sólo haber nombrado a los más destacados sino también a los que se corresponden mejor con el perfil que enunciaré.

Revista de Ciencias Sociales - Número 64 (2014) - Universidad de Valparáíso - ISSN 0716-7725-Valparaíso, Chile 
uno tiene presente los lugares donde han enseñado, el prestigio intelectual con que cuentan y la difusión de sus ideas es difícil negar que constituyen una corriente hegemónica y por eso, en mi opinión, se puede hablar de ellos y sin exagerar como nuestra historia del derecho. Eric Palma ha llamado la atención que existe entre ellos un fuerte lazo y una homogeneidad temática que lleva a catalogarlos como una Escuela historiográfica ${ }^{3}$. De hecho, ellos más de una vez han ocupado la denominación de Escuela Chilena de Historiadores del Derecho para referirse a la comunidad de estudio que integran ${ }^{4}$. Si bien los elementos que pueden sostener mi postura serán expuestos más adelante comparto la observación del profesor Palma, porque entre ellos hay una organicidad que se expresa en publicaciones, organizaciones y espacios de trabajo comunes; un marco teórico e ideológico compartido; una permanencia en el tiempo considerable y una relación de discipulado evidente, con al menos tres generaciones de investigadores y finalmente (y probablemente lo más importante) unas opiniones y líneas de investigación coherentes y consistentes. Atendido lo que acabo de exponer me referiré a ellos en este trabajo con las expresiones "nuestra historia del derecho" y "Escuela Chilena de Historia del Derecho", indistintamente.

Ahora bien, respecto de su origen cabe mencionar que se encuentra en la Universidad de Chile (con la excepción de Bravo Lira, todos se formaron ahí), y en su primera etapa es posible ver, como apunta Eric Palma ${ }^{5}$, una reacción al modo de enseñar y cultivar la historia del derecho de Valentín Letelier. Manuel Salvat, al referirse a Letelier y sus discípulos, los califica como pseudo historiadores del derecho. Y en un

3. PALMA GONZÁLEZ, Eric: "Pasado, presente y futuro de la historia del derecho en Chile”, en Forum Historiae Iuris; http://fhi.rg.mpg.de/debatte/ nuovomondo/pdf\%20files/0903palma.pdf

4. El mejor ejemplo es la publicación La Escuela Chilena de Historiadores del Derecho y los estudios jurídicos en Chile, eds. Antonio Dougnac y Felipe Vicencio, 2 volúmenes, Universidad Central de Chile, Santiago, 1999.

5. PALMA, Eric: Guía de estudio $\mathrm{N}^{\circ} 12$, p.21, disponible en: http://www. ericeduardopalma.cl/centro-de-documentacion/file/14-guia-no-12.html

Facultad de Derecho y Ciencias Sociales - Universidad de Valparaíso - Chile 
párrafo resume bien las ideas de la Escuela Chilena de Historia del Derecho sobre el método de Letelier y sus discípulos, "Ni al alumno ni al jurista interesan estas elucubraciones basadas en un sociologismo trasnochado. Lo que ellos quieren saber es cuál fue el antecedente de las leyes y códigos que estudian en los ramos positivos, cuál fue y es nuestro trasfondo jurídico —el espíritu general de la legislación-y, para cumplir con este anhelo es preciso enseñarles la historia-verdad, aquella basada en documentos y datos concretos, que esté fundada en nuestra realidad histórica, que es la descendencia de lo ibérico y lo

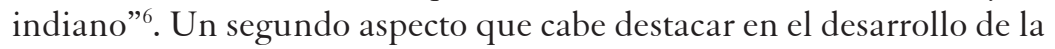
Escuela es la figura de Jaime Eyzaguirre, quien enseñó durante largos años en la Universidad de Chile y quien, a través de sus clases y su colaboración con los profesores y ayudantes de Historia del Derecho de esa casa de estudios, participó del surgimiento e influyó en la definición de los contenidos más relevantes de la Escuela Chilena de Historia del Derecho ${ }^{7}$.

A nuestra historia del derecho es posible insertarla dentro de ciertos rasgos matrices de la historiografía chilena del siglo XX, es así como Eric Palma identifica los siguientes rasgos distintivos de la "Escuela": proximidad con la historiografía conservadora del siglo XX, hispanismo y positivismo historiográfico ${ }^{8}$.

6. SALVAT MONGUILLOT, Manuel: "Necesidad de la perspectiva histórica en los estudios de Derecho”. En AA.VV., La Escuela Chilena de Historiadores del Derecho y los estudios jurídicos en Chile, eds. Antonio Dougnac y Felipe Vicencio, v. 1, Universidad Central de Chile, Santiago, 1999, p. 274.

7. Para profundizar este aspecto consultar DOUGNAC RODRÍGUEZ, Antonio: "Jaime Eyzaguirre y la Historia del Derecho". En AA.VV., La Escuela Chilena de Historiadores del Derecho y los estudios jurídicos en Chile, ob. cit., pp. 241-253 y ÁVILA MARTEL, Alamiro de: "Discurso de agradecimiento del profesor emérito Alamiro de Ávila Martel”, en Revista Chilena de Historia del Derecho, Santiago, No 15, 1989, pp. 341-350 y “Jaime Eyzaguirre y la Historia del Derecho" en Revista Chilena de Historia del Derecho, Santiago, No 5, 1969, pp. 7-8.

8. Palma también se detiene en la influencia de Alfonso García Gallo. En mi concepto las ideas del gran historiador del derecho español se proyecta en todos los ámbitos que brevemente describiré, por eso, y en aras de la brevedad, no me referiré a su influjo de manera particular (PALMA GONZÁLEZ, Eric: "Pasado, presente y futuro...”, ob. cit.).

Revista de Ciencias Sociales - Número 64 (2014) - Universidad de Valparáiso - ISSN 0716-7725-Valparáís, Chile 
En cuanto a la pertenencia a la Escuela Conservadora uno puede rastrearla por tres vías. En primer término, autores chilenos han mencionado a miembros de nuestra historia del derecho como historiadores conservadores 9 . En seguida, si la Historiografía Conservadora tiene una idea madre ésta no es otra que aquella que sostiene que el argumento central de la historia de Chile es el 'orden en sî' fundado por el gran estadista Portales ${ }^{10}$. Esta corriente intelectual, que ha construido - para algunos autores - un cierto sentido común desde la difusión de sus tesis entre el público culto ${ }^{11}$, configuró una idea hegemónica sobre el significado de la obra del célebre ministro. Así es como su herencia más fuerte y duradera, el "mito portaliano", está presente en las obras más relevantes de varios miembros de la Escuela Chilena de Historia del Derecho. Es el caso de Alamiro de Ávila, quien al referirse a la situación de Chile posterior a 1833 y la modificación que había experimentado la convivencia social después de los ensayos liberales sostiene que ese cambio no se explicaba a través de la nueva Constitución "sino el[al] espíritu insuflado al régimen por Portales: un total respeto a la autoridad, una administración limpia y

9. Respecto de Bernardino Bravo Lira, ver "Balance historiográfico y una primera aproximación al canon”. En AA.VV., Balance Historiográfico Chileno. El orden del discurso y el giro crítico actual, ed. Luis de Mussy, Ediciones Universidad Finis Terrae, Santiago, 2007, p. 52. Sobre Alamiro de Ávila, ver GAZMURI, Cristián: La Historiografía Chilena (1842-1970), Tomo II, Centro de Investigaciones Barros Arana, Taurus, 2009, p. 207 y p. 209. Se puede consultar también: GARCÍA-HUIDOBRO BECERRA, Cristóbal, Recensión al trabajo de Gabriel Salazar "Construcción de Estado en Chile (1800-1837). Democracia de los "pueblos”, militarismo ciudadano. Golpismo oligárquico”. En: Revista Historia, No 40, Vol. I, p. 196.

10. SALAZAR, Gabriel: Construcción de Estado en Chile (1800-1837). Democracia de los "pueblos". Militarismo ciudadano. Golpismo oligárquico, Editorial Sudamericana, $2^{\text {a }}$ edición, Santiago, 2007, p. 32.

11. CORVALÁN MÁRQUEZ, Luis: "Notas preliminares para un estudio sobre la relación entre historiografía y política en el pensamiento conservador chileno”. En Encuentro XXI, año 4, No 13, 1998, pp. 116. La misma idea se puede ver reiterada en JOCELYN-HOLT LETELIER, Alfredo: "Balance historiográfico y una primera aproximación al canon”. En AA.VV., Balance Historiográfico Chileno. El orden del discurso y el giro crítico actual, ob. cit., p. 52.

Facultad de Derecho y Ciencias Sociales - Universidad de Valparaíso - Chile 
eficaz, lo que permitió la tranquilidad pública, el desarrollo económico y cultural y la alta respetabilidad del país en el exterior" ${ }^{12}$. Asimismo, encontramos exposiciones similares en obras de Bernardino Bravo Lira ${ }^{13}$. En todo caso, juicios de indudable talante conservador son fáciles de encontrar en sus trabajos u opiniones. Por ejemplo, la recomendación hecha en 2005 a la Unión Europea de designar un monarca que guíe sabiamente sus destinos ${ }^{14}$ o hablar de mestizos de segundo orden, "desprovistos de elementos valóricos marcados"15. Hay, además, una evidencia de tipo institucional que agregada a los otros ayuda a ubicarlos en una determinada corriente: todos sus miembros más destacados fueron o son integrantes de la Academia Chilena de Historia, dependiente del Instituto de Chile ${ }^{16}$.

12. ÁVILA MARTEL, Alamiro de: Andrés Bello. Breve ensayo sobre su vida y su obra, Editorial Universitaria, $2^{a}$ edición, Santiago, 1987, p. 41.

13. BRAVO LIRA, Bernardino: "Portales y el tránsito del Absolutismo Ilustrado al Estado constitucional de Chile”. En AA.VV., Portales, el hombre y su obra. La consolidación del gobierno civil, comp. Bernardino Bravo Lira, Editorial Jurídica de Chile, Santiago, 1989, pp. 438 y ss.

14. BRAVO LIRA, Bernardino: "In necessariis unitas. Constitución europea, traje a la medida de un continente”. En Estudios Públicos, N 98, 2005.

15. DOUGNAC RODRÍGUEZ, Antonio: “Mestizos de segundo orden”, Derecho Mayor, en el cuerpo "Reportajes" de El Mercurio, 4 de septiembre de 2011.

16. Para un miembro de la Academia (el profesor Cristián Gazmuri) ésta tiene un indiscutible cariz conservador. El mencionado profesor indica una serie de símbolos que confluyen en su fundación evidenciando el talante conservador que la inspira: primero, nació bajo el auspicio de la Universidad Católica de Chile; en vísperas del centenario de la Constitución de 1833 y además, surgió como una alternativa a la Sociedad Chilena de Historia y Geografía, de tendencia laica y liberal. Agrega Gazmuri que para él la inspiración más definida de la Academia era su comunión con una postura definida: la cristiana e hispanista. Su secretario fue, por más de 30 años, Jaime Eyzaguirre. La Academia publica desde su fundación un Boletín de la Academia Chilena de Historia, que se transformó — siempre en la opinión de Cristián Gazmuri- en el órgano de difusión de una línea historiográfica y de un grupo de historiadores más bien cerrado (GAZMURI, Cristián: ob. cit., pp. 98 y ss.).

Revista de Ciencias Sociales - Número 64 (2014) - Universidad de Valparaíso - ISSN 0716-7725-Valparaíso, Chile 
En los trabajos historiográficos, el hispanismo suele asociarse al conservadurismo y, en efecto, normalmente van unidos. $\mathrm{Y}$ nuestros historiadores del derecho parecen no ser la excepción, esta es una idea que ha sido presentada con fuerza por Eric Palma, aunque Carlos Peña ${ }^{17}$ observó la misma circunstancia años antes. Aun a riesgo de decir cosas muy obvias hay que recordar que frente a la posición de la historiografía liberal del siglo XIX, sumamente crítica del período de dominación española, apareció en Chile una corriente opuesta, el hispanismo, que tuvo en Jaime Eyzaguirre su principal exponente y que revalorizó el legado cultural y religioso del pasado hispánico, en el que vio el elemento central de la identidad histórica del país. Esa labor intelectual se orientó tanto a la superación de la leyenda negra de la Conquista como a presentar una interpretación del proceso de independencia diverso del que hasta ese momento se conocía y manejaba ${ }^{18}$. En los trabajos de nuestra historia del derecho no cuesta encontrar afirmaciones de admiración hacia España. Por ejemplo, Manuel Salvat argumenta con fuerza, defendiendo la relevancia que el derecho español tuvo en la formación de nuestro Código Civil, atribuyendo la negación de este hecho a la leyenda negra contra España ${ }^{19}$. Del mismo modo, Antonio Dougnac al referirse a su profesor Jaime Eyzaguirre recuerda "para todos los que habíamos recibido en nuestras clases secundarias la permanente monserga del abuso de los españoles, de las clásicas causas de la independencia y de tanta banal repetición del pensamiento histórico decimonónico, las palabras del maestro resultaban un cambio radical en la apreciación de los acontecimientos" ${ }^{20}$. Y de nuevo Dougnac menciona a Alamiro de Ávila y Manuel Salvat como "campeones" en

17. PEÑA GONZÁLEZ, Carlos: "Hacia una caracterización del Ethos legal: De nuevo sobre la cultura jurídica”. En: Evolución de la cultura jurídica chilena, CPU, Santiago, 1994, p. 48.

18. Explicado fundamentalmente en EYZAGUIRRE, Jaime: Ideario y ruta de la emancipación chilena, Editorial Universitaria, Santiago, 25ª edición, 1998.

19. SALVAT MONGUILLOT, Manuel: “Necesidad...”, ob. cit., p. 265.

20. DOUGNAC RODRÍGUEZ, Antonio: “Jaime Eyzaguirre...”, ob. cit., p. 243.

Facultad de Derecho y Ciencias Sociales - Universidad de Valparaíso - Chile 
la divulgación del devenir de la Madre Patria y su vinculación con las Indias $^{21}$. Finalmente, el considerar, como lo hacía Eyzaguirre, al hispanismo como la veta central de nuestra cultura, llevó a nuestra historia del derecho a dirigir buena parte de su actividad investigadora a la comprensión de los elementos formativos de nuestro derecho: el derecho indiano y el castellano medieval, ya que ahí se encuentra gran parte de la valiosa herencia que nos dejó España.

En cuanto al positivismo historiográfico, explica bien lo que la "Escuela" entiende por tal Francisco Tomás y Valiente cuando indica que el historiador adopta "intencionalmente una actitud aséptica ante la realidad estudiada”, el investigador no pasa de ser un mero receptáculo de lo que las fuentes dicen, no opina, no valora: describe las fuentes y transmite sus noticias. Hay, entonces, en el historiador un voluntario vaciamiento de toda carga ideológica, que se entiende como propio de una actitud objetiva en el trabajo científico ${ }^{22}$. Así, ellos entienden su trabajo como el registro y la exposición sobria de (algunos) hechos, lo central en su labor historiográfica no es la interpretación sino una exposición detallada y políticamente neutral. Obviamente ideas tan ingenuas como esas se pueden cuestionar, pero mi intención es hacer una pequeña revisión de sus características, no formular una crítica epistemológica. En cualquier caso, la forma correcta de criticarlos en ese terreno es evidenciar sus contradicciones (no hacer una objeción en abstracto), porque precisamente éstas dejan entrever sus convicciones y lo ilusorio de un trabajo completamente libre de valores.

Nuestros historiadores varias veces explicitan su adhesión a la idea de que la forma correcta de hacer historia es la que acabamos de exponer. Pero nos concentraremos en dos opiniones más que importantes. Alamiro de Ávila se ubica en el bando positivista cuando

21. DOUGNAC RODRÍGUEZ, Antonio: "Alamiro de Ávila Martel cofundador de la Escuela Chilena de Historiadores del Derecho". En AA.VV., La Escuela

Chilena de Historiadores del Derecho y los estudios jurídicos en Chile, eds. Antonio Dougnac y Felipe Vicencio, t. 1, Universidad Central de Chile, Santiago, 1999, pp. 237 y 238.

22. TOMÁS Y VALIENTE, Francisco: Manual de Historia del Derecho español. Editorial Tecnos, Madrid, 1981, p. 65.

Revista de Ciencias Sociales - Número 64 (2014) - Universidad de Valparáíso - ISSN 0716-7725-Valparaíso, Chile 
afirma que José Toribio Medina es el pater familias de nuestros investigadores en el campo de la Historia ${ }^{23}$, y también cuando recordando la conocida disputa entre Lastarria y Andrés Bello critica la "tendencia filosófica" del primero que se caracterizaba por pretender hacer grandes construcciones sin estudiar previamente las fuentes. Ávila Martel toma partido por Andrés Bello quién sentó la "buena doctrina" de que era necesario estudiar los documentos, criticarlos y exponer claramente los resultados de la investigación y sólo después de esto arriesgarse a emitir juicios ${ }^{24}$. Por su parte, Bravo Lira ubica tres pasos en el método histórico: primero la búsqueda de los testimonios, luego la crítica de esos testimonios para determinar su veracidad y, por último, está la reconstrucción de lo que pasó ${ }^{25}$. Fortalece sus afirmaciones recurriendo a Von Ranke ${ }^{26}$ quien "fija el objeto de la historia en la exposición de los hechos tal como sucedieron”27. De no ser así, para Bravo Lira, el investigador proyecta sus propios criterios a la época estudiada y enjuicia, arbitrariamente, los hechos pasados.

En todo caso, su postura no escapa a la tendencia general de la historiografía universitaria chilena durante buena parte del siglo XX:

23. ÁVILA MARTEL, Alamiro de: Curso de Historia del Derecho, tomo I, Editorial Jurídica de Chile, Santiago, 1955, p. 79.

24. ÁVILA MARTEL, Alamiro de: Curso..., ob. cit., p. 77. Ávila Martel se centra en el trabajo leído en 1844 por Lastarria titulado Investigaciones sobre la influencia social de la conquista y del sistema colonial de los españoles en Chile. Para Ávila Martel la obra es una "diatriba declamatoria y no documentada contra el sistema español”. Esta obra sirvió — según Ávila Martel— para remover el ambiente en el tema historiográfico.

25. BRAVO LIRA, Bernardino: Historia de las Instituciones políticas de Chile e Hispanoamérica, Editorial Andrés Bello, 2a edición, Santiago, 1993, p. 18.

26. Leopoldo Von Ranke (1795-1886) fue un célebre historiador alemán, considerado como fundador de la ciencia histórica, su labor — definida fuertemente por el positivismo - se caracterizó por la centralidad del análisis de las fuentes primarias, principalmente documentos oficiales y por el uso del método narrativo. Para Ranke lo más importante era establecer los hechos excluyendo las interpretaciones, dejando al pasado hablar a través de los documentos.

27. Ibíd., p. 19.

Facultad de Derecho y Ciencias Sociales - Universidad de Valparaíso - Chile 
concentrarse en los tiempos lejanos, "despegarse de las peligrosas ciencias sociales" y convertir a Barros Arana o José Toribio Medina en el paradigma historiográfico a imitar y reproducir ${ }^{28}$. Aunque refugiarse en el positivismo traiga consigo "una suerte de mitologización y fetichización de los datos, los archivos, las fuentes y los métodos" ${ }^{\text {. }}$.

Además, junto a estos rasgos yo agregaría una cuarta característica. En mi opinión esta sería un vínculo especial con la tradición. Desde mi punto de vista este elemento es definitorio y distintivo de nuestra historia del Derecho. Y para conceptualizarla es conveniente recurrir a la distinción entre tradicionalismo y pensamiento conservador que, como se sabe, formuló Karl Mannheim ${ }^{30}$. Para este destacado pensador la primera actitud se caracteriza por ser una tendencia inconsciente (y latente) a preferir el pasado a lo nuevo, una determinada actitud frente al cambio social. Existe en la Escuela Chilena de Historia del Derecho una valoración de la tradición que a mi juicio se proyecta en varias cuestiones relevantes, pero la más importante es su relación con el oficio de historiador y el objeto de estudio. Una buena descripción nos las da Alamiro de Ávila, cuando recordando a Jaime Eyzaguirre dice que junto a él y otros se dedicaron a "una recreación del pasado jurídico" 31 , lo que indica una forma especial de entender su oficio y su función. Pero probablemente el mejor ejemplo lo constituyen algunos de los trabajos prosopográficos sobre grandes juristas indianos de Javier Barrientos ${ }^{32}$. Este gusto por la tradición no lo entiendo,

\footnotetext{
28. SALAZAR, Gabriel: ob. cit, p. 34.

29. Ídem.

30. MANNHEIM, Karl: "Conservative Thought". En From Karl Mannheim, ed. Kurt Wolff, Nueva York, Oxford University Press, 1971.

31. ÁVILA MARTEL, Alamiro de: “Jaime Eyzaguirre...”, ob. cit., p. 7.

32. Como ejemplos los trabajos "Juan Francisco Montemayor de Cuenca (16181685) entre Derecho indiano, Derecho común y Derecho foral”. En Revista de Estudios Histórico-Jurídicos, $\mathrm{N}^{\circ}$ 23, Valparaíso, pp. 125-208 y "Melchor Bravo de Sarabia (1512-1577): Primer Miembro del Colegio de San Clemente de Bolonia en la Judicatura Indiana, y su Familia de Togados”. En Revista de Estudios Histórico-Jurídicos, $\mathrm{N}^{\circ}$ 29, Valparaíso, pp. 423-436.
}

Revista de Ciencias Sociales - Número 64 (2014) - Universidad de Valparáíso - ISSN 0716-7725-Valparáiso, Chile 
siguiendo a Mannheim, como una opción deliberada por la preservación de un orden económico, social y político anterior (que constituiría una actitud conservadora), sino que más bien es una predilección por lo que nos ha precedido, por seguir vinculado con el pasado invocándolo, cuidando ese patrimonio, evitando que desaparezca e identificándose con él.

\section{Nuestra historia del derecho y el Derecho indiano}

En este acápite pretendo exponer brevemente qué caracterizaba al ordenamiento jurídico colonial como también los términos de la relación entre el derecho indiano y la Escuela Chilena de Historia del Derecho. Ambas cuestiones son centrales para la comprensión del resto del trabajo.

El Derecho Indiano fue el ordenamiento jurídico que rigió las colonias del Imperio Español entre los siglos XV y XIX ${ }^{33}$. Estaba formado por el derecho castellano aplicado en las Indias, los derechos indígenas y el derecho específico de Indias, este último se aplicaba de manera preferente (como derecho propio) y la normativa castellana cumplía el rol de derecho supletorio. El sistema normativo colonial era muy complejo, porque no tenía como eje un sistema de concentración absoluta del poder como sería desarrollado por el Estado moderno y porque reconocía la diversidad de la amplia extensión del Imperio. Estas características (y otras) generaron un sistema abierto a la equidad, con diversos centros de producción legislativa y con una actividad de adjudicación flexible, en la que eran admitidas y se recurría a todas las fuentes del derecho (leyes, costumbre, jurisprudencia, equidad, etc.).

33. Sobre esta materia hay una enorme bibliografía de altísima calidad, sólo destacaré las obras que yo he consultado: TAU ANZOÁTEGUI, Víctor: Casuismo y Sistema, Instituto de Investigaciones de Historia del Derecho, Buenos Aires, 1992 ; DOUGNAC RODRÍGUEZ, Antonio: Manual de Historia del Derecho Indiano, Universidad Nacional Autónoma de México, México, 1994; BRAVO LIRA, Bernardino: Derecho común y derecho propio en el nuevo mundo, Editorial Jurídica de Chile, Santiago, 1989; FIGUEROA QUINTEROS, María Angélica: “La codificación civil chilena y la estructuración de un sistema jurídico legalista”. En AA.VV., Andrés Bello y el Derecho, Editorial Jurídica de Chile, Santiago, 1982.

Facultad de Derecho y Ciencias Sociales - Universidad de Valparaíso - Chile 
En este sistema el rol central le cabía al juez, éste determinaba el derecho aplicable a cada caso. Una cuestión relevante y no siempre destacada es aclarar que la función jurisdiccional era cumplida por los corregidores y los alcaldes ordinarios en primera instancia; el cabildo, por su parte, actuaba en algunos casos como tribunal de apelación y la Real Audiencia constituía un tribunal de segunda instancia. Es necesario mencionar esto porque eran órganos que también cumplían labores de gobierno así como de producción de normas jurídicas generales. Por ello era inevitable que, al resolver litigios, su razonamiento no fuese estrictamente normativo, y que se consideraran argumentos de conveniencia y se resolvieran los conflictos considerando las consecuencias sociales de la decisión, explicando así la flexibilidad y creatividad con que se resolvían las disputas ya que no eran cuerpos concentrados exclusivamente en funciones judiciales. Todo esto favorecía una relación entre derecho y sociedad mucho más estrecha, y obviamente alejada de la idea (posterior por cierto) del Derecho como una entidad autónoma y cerrada.

Pero la singularidad del Derecho Indiano no termina ahí, porque un sistema jurídico como el que he descrito también tenía una enseñanza del derecho distinta a la que conocemos, en ella lo central era comprender los cimientos del edificio jurídico y las clases se estructuraban a partir de las exposiciones de los alumnos y se ponía énfasis en el diálogo y la discusión. Lo que se perseguía era desarrollar en el futuro letrado una formación en destrezas jurídicas que le permitieran buscar una solución original, argumentando de forma convincente y sabiendo combinar los materiales que provenían de la costumbre, jurisprudencia o doctrina. La formación incluía una pasantía en el estudio de un abogado, y un examen en el que se exponía y resolvía un pleito. Finalmente, la descripción del derecho indiano no estaría completa si no se destaca que la práctica jurídica favorable a la interpretación y adaptación del derecho se fundaba en la idea de que las normas de derecho debían estar fundadas en la razón y el bien común o de lo contrario perdían su naturaleza, concepción postulada por la axiología jurídica iusnaturalista que fue impuesta en las Indias.

Ahora, en cuanto al segundo punto, esto es, la relación entre la Escuela Chilena de Historia del Derecho y el derecho indiano, éste no

Revista de Ciencias Sociales - Número 64 (2014) - Universidad de Valparáíso - ISSN 0716-7725-Valparaíso, Chile 
es sólo su objeto de investigación predilecto (y donde han hecho sus mayores y mejores aportes) sino también es posible identificar una admiración por el mencionado ordenamiento que subyace en la mayoría de sus trabajos, pese a su compromiso con presentar el derecho "con objetivo realismo, sin ideologías a priori" ${ }^{34}$ constituye la proyección más prístina del (supuesto) hispanismo de la Escuela. Es así que el profesor Sergio Martínez Baeza ubica el entusiasmo con el derecho indiano dentro de un plan mayor, al agradecer a grandes estudiosos de la historia del derecho por "destacar los aspectos positivos de la colonización española de nuestra América”, entre los que se cuentan la fe cristiana y la lengua de Cervantes, junto con "la genial concepción del Derecho Indiano, como proyección ultramarina de aquel viejo Derecho Castellano" ${ }^{35}$.

Trataremos en las líneas siguientes de aclarar cómo se expresa esa admiración y en qué campos se proyecta. Para empezar, citaremos las palabras de Alamiro de Ávila sobre el sistema jurídico castellanoindiano. Según el profesor éste "tiene una básica inspiración en las normas del derecho natural católico. Esto lleva a considerar en las fuentes del derecho la prioridad del derecho natural sobre la ley positiva lo cual tiene muchas consecuencias, entre ellas el arbitrio judicial, que no es arbitrariedad sino atribución para crear normas más adaptadas a las circunstancias que aquellas contenidas en los textos legales y, por lo tanto, la posibilidad de enervar la ley injusta. Pero además en el sistema mismo de la producción legislativa, constantemente se concretan normas de raigambre moral y se considera que la autoridad tiene el deber de intervenir en la vida de los individuos para obligarlos a actuar rectamente" ${ }^{16}$. Este párrafo relativamente extenso se transcribe porque

34. ÁVILA MARTEL, Alamiro de: "Discurso de agradecimiento del profesor emérito Alamiro de Ávila Martel”, en Revista Chilena de Historia del Derecho, Santiago, No 15, 1989, p. 342.

35. MARTÍNEZ BAEZA, Sergio: "El derecho castellano-indiano en el Código Civil de Bello". En Revista Chilena de Historia del Derecho, N² 20, 2008, p. 366 .

36. ÁVILA MARTEL, Alamiro de: "Régimen jurídico de los trabajadores de las minas al final del periodo indiano en Chile". En Estudios de Derecho del Trabajo $y$ de Seguridad Social ofrecidos a Don Francisco Walker Linares, Editorial Jurídica, Santiago, 1968, p. 37.

Facultad de Derecho y Ciencias Sociales - Universidad de Valparaíso - Chile 
resume muy bien el enfoque de la Escuela Chilena de Historia del Derecho respecto del derecho indiano. A partir de lo que señala Ávila Martel se pueden enumerar los aspectos que nuestra historia del derecho destaca como definitorios del Derecho indiano y que, además, aprueba con entusiasmo. Está la inspiración en las normas del derecho natural, característica también expresada por Sergio Martínez cuando dice que el derecho indiano "se encuentra íntimamente vinculado a la moral cristiana y al derecho natural" ${ }^{37}$; el segundo componente es el arbitrio judicial que permite crear normas adaptadas a la cambiante realidad de territorios además muy diversos entre sí; relacionado con los aspectos anteriores está la posibilidad de suspender la aplicación de la ley si ésta se estima injusta por el juzgador.

Si bien la cita de Ávila Martel se concentra en el nervio de la relación entre la Escuela Chilena de Historia del Derecho y el derecho indiano hay elementos que no menciona pero que sí están destacados por integrantes de la Escuela. En primer término, autores como Javier Barrientos o Antonio Dougnac mencionan el "derecho al buen gobierno", del cual eran titulares "los vasallos de la monarquía hispanoindiana" ${ }^{38}$. Así, como señala Barrientos, éstos no se encontraban desamparados frente a las actuaciones de sus gobernantes, ni menos entregados al arbitrio de sus agentes ${ }^{39}$. Esta prerrogativa, además, disponía de un instrumento jurídico para hacerse exigible: la apelación gubernativa, que constituyó "el más eficaz remedio para proteger los derechos de la persona en el Estado Indiano frente a la actuación de los gobernantes" ${ }^{40}$. Por medio de esta prerrogativa (y su cautela a través del mencionado instrumento) se evitaba que las autoridades se salieran de los cauces que la ley y la costumbre fijaban ${ }^{41}$. En segundo lugar, nuestra

\author{
37. MARTÍNEZ BAEZA, Sergio: “El derecho...”, ob. cit., p. 361. \\ 38. BARRIENTOS GRANDÓN, Javier: “La fiscalización...”, ob. cit, p. 105. \\ 39. Ídem. \\ 40. Ídem. \\ 41. DOUGNAC RODRÍGUEZ, Antonio: Manual..., ob. cit., p. 383.
}

Revista de Ciencias Sociales - Número 64 (2014) - Universidad de Valparáíso - ISSN 0716-7725-Valparaíso, Chile 
historia del derecho ha desarrollado la idea de que existió, en el período de dominación española, un potente estatuto protector del indígena. Los aborígenes — según la Escuela Chilena de Historia del Derechoeran objeto de preocupación y cuidado por parte de la Corona, ya que, por ejemplo, se respetaba su derecho consuetudinario. Asimismo, se destacan los privilegios en materia procesal, en la existencia de la figura de un protector de indios y que se les considerara incapaces relativos porque eso los hacía objeto de la máxima protección por la autoridad ${ }^{42}$.

Respecto de lo que acabo de decir, me interesa destacar lo que sigue. Primero, en torno a la relación de la Escuela Chilena de Historia del Derecho con el ordenamiento indiano mostrar la admiración que aquella siente por éste, espero que haya quedado claro. En segundo lugar, y combinando lo señalado en los párrafos anteriores con la descripción que previamente hicimos del orden jurídico colonial, parece obvio que el Derecho indiano tenía un programa que cumplir, no era un derecho definido únicamente en base a formas, sino que tiene finalidades sustantivas. Este objetivo está bien resumido por un miembro de la Escuela Chilena de Historia del Derecho al decir que el derecho indiano "procura hacer carne los principios católicos que han informado a su predecesor, el castellano, desde sus orígenes” ${ }^{43}$.

\section{El tratamiento del Código Civil por nuestra historia del derecho}

En relación con el Código Civil (y en línea con su enfoque sobre el quehacer de la historia del derecho) la Escuela Chilena de Historia del Derecho se ha preocupado por exponer, detalladamente, la cronología del proceso de configuración del Código Civil, indicando las distintas etapas de su redacción, los pasos iniciales, la preparación del Código y sus fuentes. Ejemplos son el trabajo de Jaime Eyzaguirre ${ }^{44}$

42. Para profundizar estas ideas ver SALVAT MONGUILLOT, Manuel: "Los representantes de la República”. En Revista Chilena de Historia del Derecho, $\mathrm{N}^{\circ} 6,1970$, pp. 124 a 128.

43. DOUGNAC RODRÍGUEZ, Antonio: Esquema del Derecho de Familia indiano, Ediciones del Instituto de Historia del Derecho Juan de Solórzano y Pereyra, Santiago, 2003, p. 427.

44. EYZAGUIRRE, Jaime: Historia del Derecho, Editorial Universitaria, Santiago, 1991, $11^{\text {a }}$ edición, pp. 207 a 210.

Facultad de Derecho y Ciencias Sociales - Universidad de Valparaíso - Chile 
o, con mayor desarrollo y profundidad, el de Antonio Dougnac ${ }^{45}$. Por otro lado, la cerrada preponderancia de la ley como fuente del derecho ${ }^{46}$ es sólo un elemento, que cabe incluir en la descripción junto a otros rasgos. También por la vía de realizar estudios biográficos sobre Andrés Bello, o sobre su filosofía jurídica han aportado a la comprensión de nuestro proceso de codificación civil.

Hasta aquí su trabajo guarda perfecta congruencia con lo que uno esperaría de ellos. No obstante sí han formulado una idea original en la que vienen insistiendo hace ya muchos años y que aparentemente se inscribe en la línea de desmitificar creencias equivocadas de los operadores jurídicos utilizando la historia del derecho. Esta idea es el argumento central en su estudio del Código civil chileno y viene a contradecir un "lugar común" de nuestra cultura jurídica. Este lugar común sería la idea (errada) de considerar que el Código de Bello es en sus aspectos centrales (de contenido y sistemáticos) una reproducción del Código Civil francés, que sería la principal fuente de inspiración del cuerpo legal redactado por Andrés Bello.

La tesis de la Escuela Chilena de Historia del Derecho es otra, para sus integrantes lo que se codificó fue el derecho castellano indiano. Bravo Lira, por ejemplo, dice que fundamentalmente "la labor de Bello consistió en formar un código de Derecho nacional, sin aventurarse a copiar otro extranjero, ni menos probar soluciones propias (...) Lo que hizo fue reformular el derecho castellano que regía en Chile y en América española, en un texto tan elegante como preciso" ${ }^{47}$. Dicho de forma más breve, por Alamiro de Ávila "el Código Civil no salió de la mente del codificador, sino que existía con anterioridad. $\mathrm{Y}$ era válido

45. DOUGNAC RODRÍGUEZ, Antonio: "La codificación civil en Chile y la formación de su “Código Civil’”. En AA.VV., El Código Civil de Chile (18552005), Lexis Nexis, Santiago, 2007, pp. 135-150.

46. ÁVILA MARTEL, Alamiro de: Andrés Bello..., ob. cit., p. 64.

47. BRAVO LIRA, Bernardino: "Puntales de la codificación en el Viejo y en el Nuevo Mundo (1797-1855). Tres grandes modelos: Von Martini en Austria, Portalis en Francia y Bello en Chile”, En AA.VV., El Código Civil de Chile (18552005), Santiago, Lexis Nexis, 2007, p. 125.

Revista de Ciencias Sociales - Número 64 (2014) - Universidad de Valparáíso - ISSN 0716-7725-Valparaíso, Chile 
en toda América”" ${ }^{\$ 8}$. Esta es la idea central, no hubo novedad en el Código Civil porque si bien su forma es decimonónica (la técnica sistemática y cerrada de la ley) el fondo es indiano. De Andrés Bello entonces cabe destacar su capacidad para "aprovechar el inmenso bagaje jurídico — sobre todo, legal y doctrinario- aportado al mundo hispanoamericano por la tradición castellana, enriquecida con no pocas contribuciones indianas (...) para combinarlo con los logros modernos de las codificaciones hasta entonces existentes" ${ }^{49}$. Por ello Javier Barrientos habla de un código civil imaginario, aquél que fundado en opiniones poco documentadas identifica el Código Napoleón con el Código Civil chileno, creando problemas también imaginarios que se resolverían sin dificultad si la solución deja de buscarse en el derecho francés y se atiende al origen castellano de las normas ${ }^{50}$.

\section{La primera contradicción de nuestra Historia del Derecho}

Antes que todo cabe una prevención, esta contradicción es la más importante, sobre la que me extenderé y las siguientes contradicciones son, en buena parte, una proyección y se encuentran fundadas en la que a continuación explicaré. Lo que intentaré demostrar aquí es que la interpretación que la Escuela Chilena de Historia del Derecho hace del Código Civil chileno y su origen lleva a sus integrantes a contradecirse, porque terminan valorando dos sistemas jurídicos incompatibles entre sí.

Primero, de lo dicho a propósito del análisis que la Escuela Chilena de Historia del Derecho hace del Código Civil, se puede extraer

48. ÁVILA MARTEL, Alamiro de: "Bello y el Derecho Romano". En AA.VV., Estudios sobre la vida y obra de Andrés Bello, Ediciones de la Universidad de Chile, Santiago, 1973, p. 96.

49. Ibíd., p. 150.

50. BARRIENTOS GRANDÓN, Javier: "De las voces 'tradición' y 'entrega' en el Código Civil Chileno. Sobre la civilística chilena y nuestro código civil imaginario I". En AA.VV., Estudios de Derecho Romano en Homenaje al Prof. Dr. D. Francisco Samper Polo; ed. Patricio Carvajal, Librotecnia, 2007, p. 135.

Facultad de Derecho y Ciencias Sociales - Universidad de Valparaíso - Chile 
la idea de que el Código Civil no marca la supresión del derecho indiano; lo que habría tenido lugar es una simple reformulación del derecho precedente, o sea, del contenido del derecho indiano que se correspondía con el espíritu de nuestra nación. Por lo tanto, así pervive el derecho indiano, y a través de él, la normativa castellana. Se le reconoce a Andrés Bello el gran mérito de reformular el derecho vigente a la época de la redacción del Código, sin pretender agregar cuestiones propias o trasplantar instituciones foráneas. Así lo apunta Alamiro de Ávila: “el derecho privado de los diversos países de América, en la época indiana, con muy pocas peculiaridades, era uno mismo y lo que Bello hizo fue, en la mayor parte del texto legal, poner en artículos, en la técnica de la codificación francesa, el derecho que a la sazón era el vigente" ${ }^{51}$. De este modo se puede sostener que ese derecho indiano se presentó ocupando la estructura de la codificación, con normas formuladas claramente y fáciles de aplicar, sin las glosas contradictorias de los juristas, quitándole el marasmo reglamentario.

En suma, la forma cambia, muda la manera de presentar las disposiciones, pero el fondo no cambia. En un nuevo molde que aporta certeza y seguridad jurídica para el tráfico privado, el Código Civil presentó el mismo contenido probado ya por siglos en los dominios americanos de la Corona española. De este modo, no abrazan la codificación porque sea algo nuevo, la abrazan porque en realidad es algo viejo con lo que se sienten sumamente cómodos.

Pero la verdad es que se equivocan, en el Código Civil no hay pervivencia de lo indiano y ello por varias razones.

Primero, la afirmación que sostiene que Andrés Bello recogió el derecho vigente ${ }^{52}$ constituye un error porque lo que distinguía al derecho indiano era su flexibilidad, su capacidad de adaptación, que se correspondía con la idea que las normas eran una forma de

51. ÁVILA MARTEL, Alamiro de: "Bello y el Derecho Romano", ob. cit., p. 96.

52. En cualquier caso, la idea de que Andrés Bello recogió el derecho indiano vigente es sólo parcialmente correcta, ya que la conclusión más extendida en nuestra doctrina es que en materia de familia el contenido es indiano, mas no así en materia de obligaciones o bienes (TAPIA RODRÍGUEZ, Mauricio: Código Civil 1855- 2005. Evolucion y perspectivas, Editorial Jurídica, 2005, Santiago, p. 32)

Revista de Ciencias Sociales - Número 64 (2014) - Universidad de Valparaíso - ISSN 0716-7725-Valparáís, Chile 
aproximación a la equidad natural, y por ello, lo fundamental era encontrar la solución justa. No se condice con el "espíritu” del sistema indiano fijarlo de una vez y por mucho tiempo. En este orden de ideas bastaría ver los primeros artículos del título preliminar del Código Civil que son, la antítesis de las ideas fundantes del orden indiano, para comprender el abismo que hay entre la tradición jurídica colonial y el orden de la codificación.

Asimismo, su idea de que el derecho indiano se instaló en el código civil a través de disposiciones concretas supone una concepción bien estrecha del fenómeno jurídico, para ellos el derecho no sería más que un conjunto de reglas, de enunciados que regulan la vida práctica, porque solo así se podría llegar a decir que el derecho indiano configuró el Código Civil, olvidándose de la importancia de la concepción del derecho que articula el sistema jurídico, de cómo se enseña el derecho, de lo estrecho o amplio de las potestades del juez, del rol de la comunidad en la creación jurídica. Todo eso pareciera no importar porque las disposiciones concretas perviven, pero perviven cristalizadas, fijas en una formulación del derecho que no puede estar más lejos del espíritu del derecho indiano. El derecho es una práctica social compleja donde intervienen muchísimos factores, el estudio del derecho indiano nos muestra eso y si es así no importan sólo las regulaciones concretas sino la cultura jurídica que se genera a partir de un cuerpo normativo.

Cabe también objetar la distinción entre forma y sustancia que sugiere el argumento de la Escuela Chilena de Historia del Derecho, recurriendo al jurista norteamericano Duncan Kennedy, quien en un trabajo clásico en su medio explicita ideas muy útiles para lo que intentamos explicar ${ }^{53}$. Desde ya advierto que la crítica a la idea de forma y sustancia persigue desautorizar la distinción que hace nuestra historia del derecho, pero también busca proponer una lectura que hace más profundo el error de nuestra historia del derecho.

El citado trabajo es una investigación acerca del carácter y la interconexión de los distintos modos retóricos que cabe encontrar en

53. KENNEDY, Duncan: "Forma y sustancia en la adjudicación del derecho privado”. En AA.VV., Sociología jurídica: teoría y sociología del derecho en Estados Unidos, ed. Mauricio García Villegas, Universidad Nacional de Colombia, Bogotá, 2001, pp. 162-187.

Facultad de Derecho y Ciencias Sociales - Universidad de Valparaíso - Chile 
las sentencias, artículos y tratados de Derecho Privado norteamericano ${ }^{54}$, especialmente en el Derecho de Contratos, aunque su modo de análisis puede trasladarse a otras ramas, y en general a las argumentaciones y doctrinas jurídicas dominantes ${ }^{55}$. Para explicar apretadamente cuál es la utilidad de las ideas del profesor Kennedy para este artículo hay que hacer referencia a dos distinciones. En primer lugar, y en una distinción usual en la teoría del derecho, Kennedy dice que existen dos modos opuestos de abordar la cuestión de la forma en que deben emitirse las normas o directivas jurídicas. Uno favorece el empleo de reglas generales claramente definidas. El otro favorece el uso de estándares equitativos o informales que dan lugar a decisiones ad hoc, sensibles a las circunstancias específicas de cada caso. Del mismo modo, Kennedy efectúa otra distinción. Según este autor existen también dos modos retóricos opuestos para abordar los problemas de la sustancia o contenido del Derecho, dos grandes posturas morales y políticas sustantivas: individualismo ${ }^{56}$ y altruismo ${ }^{57}$. Lo central de "Forma y sustancia..." es la idea de que las posturas divergentes en cuanto a la forma tienden a corresponder con las posturas divergentes en cuanto a la sustancia: la visión sustantiva altruista tiende a preferir los estándares flexibles, mientras que el individualismo suele asociarse más bien con el empleo de reglas rígidas.

Esta explicación tiene por objeto, en primer lugar mostrar que no se puede distinguir tajantemente entre cuestiones de forma y de

54. KENNEDY, Duncan: ob. cit, p. 163.

55. PÉREZ LLEDO: Juan Antonio: El Movimiento Critical Legal Studies, Tecnos, Madrid, 1996, p. 306.

56. El individualismo parte de una clara distinción entre los intereses de cada individuo y los de los demás, y sostiene que es legítimo anteponer nuestros intereses a los de los demás, siempre que respetemos las normas que posibilitan nuestra coexistencia con otros individuos igualmente autointeresados (PÉREZ LLEDO, Juan Antonio: ob. cit., p. 310).

57. Para Kennedy el altruismo sostiene que nuestros intereses carecen de prioridad normativa sobre los de los demás. El altruismo nos ordena hacer sacrificios, compartir y ser compasivos (KENNEDY, Duncan: ob. cit., p. 168).

Revista de Ciencias Sociales - Número 64 (2014) - Universidad de Valparáíso - ISSN 0716-7725-Valparáiso, Chile 
fondo, el problema de la forma es también un problema sustantivo. La elección de forma es rara vez puramente instrumental o táctica. Así, Kennedy dice que "la preferencia por unas reglas o criterios es un aspecto de posiciones sustantivas opuestas" ${ }^{58}$. Siguiendo esta idea, no es indiferente la forma que se ocupa para presentar el Derecho. Por ello, a partir de la relación forma/sustancia se puede ir más allá y proponer una crítica más amplia a esta contradicción en la obra de nuestra historia del derecho. Según Kennedy, hay una identificación entre contenido altruista de las disposiciones y una forma de presentar las normas en base a principios o estándares. Creo que, a partir de esa identificación (y de su opuesto, es decir, individualismo y uso de reglas), es posible aventurar que un sistema basado en reglas, como el del Código Civil, persigue - fundamentalmente - la estabilidad del intercambio económico, es decir, que éste se efectúe de la forma más predecible posible, lo que busca es certeza y seguridad. Por el contrario, un sistema que le interesa aplicar un programa altruista, como el del derecho indiano, un "derecho cristiano, amparador de los débiles, protector de la familia" ${ }^{99}$, tendería a tener más posibilidades de cumplirse a través de un esquema fundado en la existencia de principios o estándares; esto requiere para funcionar de un orden flexible de fuentes que le entregue al juez márgenes amplios de decisión. La finalidad de aplicación a cada caso de una solución justa y no estrictamente legal (una idea evidentemente altruista, porque requiere ponerse en el lugar del otro) necesita promover la creatividad judicial y reconocer valor a los estándares que pueden surgir del derecho consuetudinario. En el caso del derecho indiano la manera ( $o$, mejor dicho, las maneras) en que se expresa su normativa era determinante para que el sistema de origen castellano funcionara como esperaba. No olvidemos que perseguía unas finalidades sustantivas.

Por las razones expuestas, la Escuela Chilena de Historia del Derecho se equivoca al sostener que la base del Código Civil es el derecho hispano indiano (a menos que quede claro que con ello sólo quieran decir que las regulaciones concretas de una época de la

58. Ibíd., p. 164.

59. DOUGNAC RODRÍGUEZ, Antonio: "La unidad de domicilio conyugal en Chile indiano”. En Revista Chilena de Derecho, Vol. 7, 1980, p. 583.

Facultad de Derecho y Ciencias Sociales - Universidad de Valparaíso - Chile 
evolución indiana fueron fijadas en el Código Civil). Y ese error los lleva a contradecirse porque terminan abrazando dos sistemas jurídicos totalmente opuestos, tanto en las doctrinas de filosofía jurídica que los inspiran como en cuanto al rol del juez y los objetivos que ambos órdenes jurídicos perseguían.

\section{La segunda contradicción (o cuán hispanista es nuestra historia del derecho)}

No sólo la tesis sobre la presencia protagónica del derecho castellano indiano en el código civil muestra la defensa de lo español, también el enfoque que hace la Escuela Chilena de Historia del Derecho sobre el origen de nuestro Código Civil parece encontrar su fundamento en una interpretación realizada más de una vez por la historiografía de raíz conservadora e hispanista. Ambos datos convierten la orientación hispanista de nuestra historia del derecho en una cuestión de perogrullo. Sin embargo, quiero proponer la idea de que la tesis interpretativa está lejos de ser hispanista, al menos pensando en que lo que se buscaría reivindicar es el sustrato jurídico-cultural heredado de la Colonia.

En primer lugar, hay que explicar ese modelo de interpretación propio de la práctica historiográfica conservadora e hispanista, por medio de una idea expuesta por Alfredo Jocelyn Holt, quien presenta dos momentos centrales de nuestra evolución como país que la historiografía conservadora entiende a partir de una hebra común. En primer lugar, la independencia de Chile es entendida como un hecho meramente casual a la vez que condicionado por el acervo tradicional hispano. Para ello le basta con identificar "cierta presencia tradicional en el período, como las manifestaciones neoescolásticas en el discurso legitimista, autonomismo inicial previo a anhelos separatistas y continuidad del orden económico y social”. El segundo hito fundacional de nuestra historia es el período "portaliano" (1830-1860), caracterizado como se sabe por el dominio político de los pelucones. Nuevamente la Escuela Conservadora ve, en esta etapa de nuestro país, una restauración del "espíritu español, autoritario y tradicional”60.

60. JOCELYN - HOLT LETELIER, Alfredo: La Independencia de Chile. Tradición, Modernización y Mito, Editorial Planeta, Santiago, 1999, p. 341.

Revista de Ciencias Sociales - Número 64 (2014) - Universidad de Valparáíso - ISSN 0716-7725-Valparáiso, Chile 
A mayor abundamiento, los profesores Ávila Martel y Bravo Lira (parte de nuestra historia del derecho pero también miembros plenos de la tradición conservadora) han planteado (proyectando el enfoque recién descrito) la continuidad de la Universidad Dominica de Santo Tomás, la Real Universidad de San Felipe y la Universidad de Chile. Esta idea de Bravo Lira y Alamiro de Ávila se sostiene obviamente en una valoración intrínseca de la tradición, pero también constituye una respuesta a la tesis liberal (sustentada por Barros Arana, por ejemplo) que ve en la Universidad de Chile un quiebre con el pasado colonial. Frente a esa tesis, los historiadores del Derecho oponen otra que busca rescatar y revalorizar el legado hispánico ${ }^{61}$, repitiendo el argumento matriz de la Escuela Conservadora. La hebra común parece estar clara, el enfoque de estos hitos reconoce como nota distintiva una visión de la historia cuyo acento está puesto en el constante retorno a las raíces hispanas y católicas, siempre orientado a reivindicar una tradición y a evidenciar que lo que se disfraza como innovación o evolución no es más que la vuelta a lo español pero con otro nombre u otros ropajes.

En la misma línea se inscribe la relación de nuestra historia del derecho con el Código Civil. Como hemos podido ver en este trabajo, lo más relevante para ellos es la pervivencia de lo español en el Código Civil, esto es, se reformula algo que ya existía, se vuelve a lo que nos constituye, a nuestra veta central, y que lo que aparenta ser una novedad o es presentado como tal no es más que la actualización de nuestra tradición hispánica y católica. Ese énfasis — respetable por cierto- les impide observar con claridad que, en realidad, el Código civil (en especial su título preliminar) lo que posibilitó fue que nuestra cultura jurídica se alejara de la arquitectura jurídica indiana, y por lo tanto, de nuestra herencia española. Así las cosas, el Código Civil, más allá de recoger ordenadamente (en algunas materias) los contenidos normativos indianos, no fue un vehículo de pervivencia de la tradición colonial, sino todo lo contrario. En mi opinión aquí no sólo hay una contradicción, también hay — a la vez- una paradoja. Ésta se produce porque ocupando un método y enfoque propio de la historiografía

61. SERRANO, Sol: "La Universidad de Chile o el peso de la Historia". En Anales de la Universidad de Chile, Sexta Serie, N², diciembre de 1995, p. 67.

Facultad de Derecho y Ciencias Sociales - Universidad de Valparaíso - Chile 
conservadora e hispanista el resultado es una perspectiva incapaz de advertir cómo la tradición indiana (por lo tanto, hispana) fue sepultada por el Código Civil. Y, precisamente por ello, nuestra historia del derecho se contradice ya que invisibiliza la herencia jurídica española que admira y (asumo) pretende defender, aceptando la imposición de un modelo foráneo y ajeno a lo español.

\section{La tercera contradicción de la Escuela Chilena de Historia del Derecho (a propósito del rol de la historia del derecho)}

Existe, a mi juicio, otra contradicción, constituida esta vez por una oposición entre su idea acerca de la función de la Historia del Derecho y su trabajo efectivo en ese campo de estudio, siempre a propósito de su tesis sobre el origen del Código Civil.

Antes de plantear en qué consiste la contradicción, y como requisito indispensable para (tratar de) explicarla es necesario examinar una última arista de la Escuela Chilena de Historia del Derecho. Me refiero a su perspectiva en torno a la utilidad y sentido de cultivar y (especialmente) enseñar la Historia del Derecho. Esta se encuentra plasmada fundamentalmente en un escrito del profesor Manuel Salvat. Seguiremos ese trabajo aún cuando se incorporarán ideas de otros miembros de la Escuela Chilena de Historia del Derecho. Salvat escribió este artículo en 1967, cuando el ramo de Historia del Derecho en la Universidad de Chile había sido reducido a un semestre. El conocido profesor realiza una defensa de la Historia del Derecho justificando su relevancia y utilidad, buscando "mover a la reflexión a los tercos espíritus dogmáticos", estos espíritus son "aquellos que sienten un apego exagerado a la ley positiva, que ven en ella la única fuente de derecho y justicia posible y que, por lo tanto, estiman inoficioso el saber Derecho con perspectiva histórica" ${ }^{2}$. Al estudiar historia del derecho "se adquiere un saber que ayuda a valorar el juego de las fuentes del derecho, de las que hoy sólo se aprecia la ley parlamentaria”, ${ }^{63}$, de este modo, se conocerá

\footnotetext{
62. SALVAT MONGUILLOT, Manuel: “Necesidad...”, ob., cit., pp. 261 y 262.

63. Ibíd., p. 266.
} 
el valor de las otras fuentes del derecho en otras épocas. Así, cree Salvat "El jurista advertido en las fuentes no incurrirá en la majadería de preguntar a un estudiante qué dice el código y tendrá una saludable desconfianza acerca de la ley positiva como única norma aplicable" ${ }^{64}$.

Aunque don Manuel reconoce que nuestro sistema es de plenitud legal, llama la atención sobre una cuestión: "Si la sentencia es justa pero contraria a la ley, anulable; si se aplica una ley en que el derecho y la justicia están ausentes, lo resuelto es válido" ${ }^{\prime 5}$. Salvat tiene la esperanza de que los alumnos que se están (estaban) formando puedan influir porque el sistema legalista se mejore y cambie, para lo cual es conveniente mostrarles otros regímenes jurídicos donde hay más amplitud, más libertad y más justicia ${ }^{66}$.

Pareciera quedar claro el manifiesto de Salvat de cuáles serían las razones por las que es necesario enseñar Historia del Derecho. A través de la comprensión de ordenamientos pretéritos podemos ver que, por ejemplo, las fuentes del derecho tenían estatutos diversos, que había sistemas más abiertos que el que actualmente nos rige.

En términos parecidos se expresa Sergio Martínez Baeza, quien sostiene que mediante una perspectiva histórica el derecho adquiere una flexibilidad que lo hace más adaptable a las circunstancias externas. Si se prescinde de ésta solo se obtendrá como producto abogados convencidos de la que ley es la única forma de crear derecho, perdiendo su libertad creadora e interpretativa ${ }^{67}$.

Afirmaciones que están en consonancia con lo que otros autores han dicho, para quienes el cultivo de esta disciplina es una buena forma de relativizar lo que nos parece evidente ${ }^{68}$, convirtiéndose en un antídoto

64. Ídem.

65. Ibíd., p. 262.

66. Ídem.

67. MARTÍNEZ BAEZA, Sergio: "Escuela Chilena de Historia del Derecho: aportes y desarrollo", disponible en http://www.acaderc.org.ar/doctrina/ articulos/artescuelachilena, p. 4.

68. TOMÁS Y VALIENTE, Francisco: ob. cit., para este autor la Historia del Derecho actúa como saludable escuela de relativización (p. 34).

Facultad de Derecho y Ciencias Sociales - Universidad de Valparaíso - Chile 
para el formalismo y para quienes la perspectiva histórica "quita su carácter absoluto a las certezas actuales, las relativiza poniéndolas en fricción con certezas distintas u opuestas ya experimentadas en el pasado" ${ }^{69}$. Es difícil no compartir todas estas afirmaciones porque, sabiendo que las ordenaciones históricamente han sido distintas, eso nos permite tomar distancia y no creer que siempre nos hemos relacionado igual ni regulado nuestra convivencia de la misma manera, haciendo más compleja y rica nuestra comprensión del Derecho.

Con su argumento central de dónde encuentra sus raíces nuestro Código Civil la Escuela Chilena de Historia del Derecho hace, casi con total seguridad, exactamente lo contrario. Por razones que espero hayan quedado claras allí donde debería presentar un corte muestra continuidad y presenta esa evolución como una cuestión más o menos natural, desdibujando precisamente la perspectiva histórica.

$\mathrm{Al}$ abrazar sistemas jurídicos opuestos, al no hacer ver el contraste, lo que tiende a ocurrir es que queda la evolución de nuestro Derecho más como un continuo que como una modificación o alteración que pudo o no suceder. Y se tiende a naturalizar, a ver como algo normal y predecible un cambio que perfectamente pudo ir por otra vía, y se oculta que si se optó por un camino eso fue producto de la voluntad de ciertos sujetos. De este modo se pierde la oportunidad de poner de relieve lo relativo de nuestros ordenamientos jurídicos.

\section{Conclusiones}

El Código Civil, si lo miramos desde la perspectiva del ordenamiento vigente a su promulgación, centralizó y homogenizó el derecho cambiando su relación con la sociedad, terminó con la creatividad judicial, la apertura a la equidad y el valor de las prácticas consuetudinarias. Nuestro código civil, con todas sus virtudes, forma parte del fenómeno de la codificación y se aleja prácticamente en todo de la tradición indiana que yo no dudaría en calificar como valiosa y original. Por eso quienes aspiran a vincular ambos sistemas se equivocan.

69. GROSSI, Paolo: Mitología Jurídica de la Modernidad, Editorial Trotta, Madrid, traducción de Manuel Martínez Neira, 2003, p. 23.

Revista de Ciencias Sociales - Número 64 (2014) - Universidad de Valparaíso - ISSN 0716-7725-Valparaíso, Chile 
Porque al narrar que el ordenamiento colonial sobrevivió en el código civil, justificando esa tesis en una suerte de filología normativa, lo único que hacen es ocultar el monopolio de la producción jurídica, la estatización del derecho y el alejamiento de nuestras raíces hispánicas. A consecuencia de esta idea la interesante técnica indiana termina siendo silenciada, pero por una vía peligrosa, asumiendo que sobrevive en el código civil. Insistiendo en ello se termina creando un mito que se acopla perfectamente a la rutina legalista y proyectando al Derecho sólo como un conjunto de formas neutras.

Con respecto a la Escuela Chilena de Historia del Derecho, lo que cabe agregar, ya que las contradicciones han sido presentadas, es sugerir que dichas incongruencias encuentran parte de su justificación en el problema de creer en la observación neutral y en la distinción sujeto/objeto de conocimiento, y que éstas a su vez permiten una relación no interferida con la realidad ${ }^{70}$. Porque, sin importar cuáles sean esas convicciones que se declaran o no, siempre se termina haciendo reflexión académica cargada por las legítimas preferencias o creencias, pero en este caso se hace de modo inconsciente. Lo que lo hace más grave, porque es menos susceptible de algún control por el propio investigador y puede producir errores que lleven a contradecir la médula de un trabajo intelectual que en el caso de nuestra historia del derecho es precisamente la defensa del legado hispánico. Así, el llamado positivismo historiográfico deviene sólo en un discurso de justificación para conciliar artificialmente un hispanismo inconsecuente con los estándares jurídicos del statu quo legalista, liberal y positivista.

70. ARNAUD, André-Jean y FARIÑAS DULCE, María José: Sistemas Jurídicos: Elementos para un análisis sociológico del derecho, Universidad Carlos III y Boletín Ofiical del Estado, Madrid, 1996, pp. 108-109 y 114-115.

Facultad de Derecho y Ciencias Sociales - Universidad de Valparaíso - Chile 


\section{BIBLIOGRAFÍA}

ARNAUD, André-Jean y FARIÑAS DULCE, María José: Sistemas Jurídicos: Elementos para un análisis sociológico del derecho,

Universidad Carlos III y Boletín Oficial del Estado, Madrid, 1996.

ÁVILA MARTEL, Alamiro de: Andrés Bello. Breve ensayo sobre su vida y su obra, Editorial Universitaria, 2a edición, Santiago, 1987.

__ "Bello y el Derecho Romano". En AA.VV., Estudios sobre la vida y obra de Andrés Bello, Ediciones de la Universidad de Chile, Santiago, 1973, pp. 79-97.

— Curso de Historia del Derecho, Editorial Jurídica de Chile, Santiago, 1955.

__ "Discurso de agradecimiento del profesor emérito Alamiro de Ávila Martel", en Revista Chilena de Historia del Derecho, Santiago, $\mathrm{N}^{\circ} 15,1989$, p. 341-350.

__ "Jaime Eyzaguirre y la Historia del Derecho" en Revista Chilena de Historia del Derecho, Santiago, No 5, 1969, pp. 7-8.

_- "Régimen jurídico de los trabajadores de las minas al final del período indiano en Chile”. En Estudios de Derecho del Trabajo y de Seguridad Social ofrecidos a Don Francisco Walker Linares, Editorial Jurídica, Santiago, 1968, pp. 35-43.

BARRIENTOS GRANDÓN, Javier: "De las voces 'tradición' y 'entrega' en el Código Civil Chileno. Sobre la civilística chilena y nuestro

código civil imaginario I”. En AA.VV., Estudios de Derecho Romano en Homenaje al Prof. Dr. D. Francisco Samper Polo; ed. Patricio Carvajal, Librotecnia, 2007, p. 135.

—_ "La fiscalización de los actos de gobierno en la época indiana y su desaparición durante la República”. En Revista de Estudios Histórico- Jurídicos, N 15, Valparaíso, pp. 105-130.

BRAVO LIRA, Bernardino: Derecho común y derecho propio en el nuevo mundo, Editorial Jurídica de Chile, Santiago, 1989.

_- Historia de las Instituciones políticas de Chile e Hispanoamérica, Editorial Andrés Bello, 2a edición, Santiago, 1993.

_- "In necessariis unitas. Constitución europea, traje a la medida de un continente”. En Estudios Públicos, N 98, 2005.

Revista de Ciencias Sociales - Número 64 (2014) - Universidad de Valparáíso - ISSN 0716-7725-Valparaíso, Chile 
"Portales y el tránsito del Absolutismo Ilustrado al Estado constitucional de Chile". En AA.VV., Portales, el hombre y su obra. La consolidación del gobierno civil, comp. Bernardino Bravo Lira, Editorial Jurídica de Chile, Santiago, 1989, pp. 321 437.

__ "Puntales de la codificación en el Viejo y en el Nuevo Mundo (1797-1855). Tres grandes modelos: Von Martini en Austria, Portalis en Francia y Bello en Chile". En AA.VV., El Código Civil de Chile (1855-2005), Santiago, Lexis Nexis, 2007, pp. 101-133. CORVALÁN MÁRQUEZ, Luis: "Notas preliminares para un estudio sobre la relación entre historiografía y política en el pensamiento conservador chileno". En Encuentro XXI, año 4, No 13, 1998.

DOUGNAC RODRÍGUEZ, Antonio: "Alamiro de Ávila Martel cofundador de la Escuela Chilena de Historiadores del Derecho".

En AA.VV., La Escuela Chilena de Historiadores del Derecho y los estudios jurídicos en Chile, eds. Antonio Dougnac y Felipe Vicencio, t. 1, Universidad Central de Chile, Santiago, 1999, pp. 225-239.

__ Esquema del Derecho de Familia indiano, Ediciones del Instituto de Historia del Derecho Juan de Solórzano y Pereyra, Santiago, 2003.

__ "Jaime Eyzaguirre y la Historia del Derecho". En AA.VV., La Escuela Chilena de Historiadores del Derecho y los estudios jurídicos en Chile, eds. Antonio Dougnac y Felipe Vicencio, t. 1, Universidad Central de Chile, Santiago, 1999, pp. 241-253.

__ "La codificación civil en Chile y la formación de su 'Código Civil”. En AA.VV., El Código Civil de Chile (1855-2005), Lexis Nexis, Santiago, 2007, pp. 135-150.

__ "La unidad de domicilio conyugal en Chile indiano". En Revista Chilena de Derecho, Vol. 7, 1980, pp. 567-589.

_- Manual de Historia del Derecho Indiano, Universidad Nacional Autónoma de México, México, 1994.

__ "Mestizos de segundo orden”, Derecho Mayor, en el cuerpo "Reportajes" de El Mercurio, 4 de septiembre de 2011.

EYZAGUIRRE, Jaime: Historia del Derecho, Editorial Universitaria, $11^{\text {a }}$ edición, Santiago, 1991.

Facultad de Derecho y Ciencias Sociales - Universidad de Valparaíso - Chile 
- Ideario y ruta de la emancipación chilena, Editorial Universitaria, Santiago, 25 a edición, 1998.

FIGUEROA QUINTEROS, María Angélica: "La codificación civil chilena y la estructuración de un sistema jurídico legalista". En AA.VV., Andrés Bello y el Derecho, Editorial Jurídica de Chile, Santiago, 1982, pp. 77-104.

GARCÍA-HUIDOBRO BECERRA, Cristóbal: "Recensión a Construcción de Estado en Chile (1800 - 1837). Democracia de los "pueblos".

Militarismo ciudadano. Golpismo oligárquico de Gabriel Salazar”. En Historia $\mathrm{N}^{\mathrm{o}}$ 40, vol. I, 2007.

GAZMURI, Cristián: La Historiografía Chilena (1842-1970), Tomos I y II, Centro de Investigaciones Barros Arana, Taurus, 2009.

GROSSI, Paolo: Mitología Jurídica de la Modernidad, Editorial Trotta, Madrid, traducción de de Manuel Martínez Neira, 2003, p. 23.

JOCELYN HOLT, Alfredo: "Balance historiográfico y una primera aproximación al canon". En AA.VV., Balance Historiográfico Chileno. El orden del discurso y el giro crítico actual, ed. Luis de Mussy, Ediciones Universidad Finis Terrae, Santiago, 2007, pp. 31-74.

— La Independencia de Chile. Tradición, Modernización y Mito, Editorial Planeta, Santiago, 1999.

KENNEDY, Duncan: "Forma y sustancia en la adjudicación del derecho privado". En AA.VV., Sociología jurídica: teoría y sociología del derecho en Estados Unidos, ed. Mauricio García Villegas, Universidad Nacional de Colombia, Bogotá, 2001, pp. 162-187.

MARTÍNEZ BAEZA, Sergio: "El derecho castellano-indiano en el Código Civil de Bello". En Revista Chilena de Historia del Derecho, No 20, 2008, pp. 355-368.

"Escuela Chilena de Historia del Derecho: aportes y desarrollo", disponible en http://www.acaderc.org.ar/doctrina/articulos/ artescuelachilena

PALMA GONZÁLEZ, Eric: Pasado, presente y futuro de la historia del derecho en Chile, en Forum Historiae Iuris; http://fhi.rg. mpg.de/debatte/nuovomondo/pdf\%20files/0903palma.pdf

PEÑA GONZÁLEZ, Carlos: "Hacia una caracterización del Ethos legal: De nuevo sobre la cultura jurídica". En Evolución de la cultura jurídica chilena, CPU, Santiago, 1994, pp. 23-149.

Revista de Ciencias Sociales - Número 64 (2014) - Universidad de Valparáíso - ISSN 0716-7725-Valparáiso, Chile 
PÉREZ LLEDO: Juan Antonio: El Movimiento Critical Legal Studies, Tecnos, Madrid, 1996.

SALAZAR VERGARA, Gabriel: Construcción de Estado en Chile (1800-1837). Democracia de los "pueblos". Militarismo ciudadano. Golpismo oligárquico, Editorial Sudamericana, 2a edición, Santiago, 2007.

SALVAT MONGUILLOT, Manuel: "Los representantes de la República”. En Revista Chilena de Historia del Derecho, Nº 6, 1970, pp. 120-128.

_ "Necesidad de la perspectiva histórica en los estudios de Derecho". En AA.VV., La Escuela Chilena de Historiadores del Derecho y los estudios jurídicos en Chile, eds. Antonio Dougnac y Felipe Vicencio, t. 1, Universidad Central de Chile, Santiago, 1999, pp. 261-285.

SERRANO, Sol: "La Universidad de Chile o el peso de la Historia". En Anales de la Universidad de Chile, Sexta Serie, $\mathrm{N}^{\circ} 2$, diciembre de 1995, pp. 65-71.

TOMÁS Y VALIENTE, Francisco: Manual de Historia del Derecho español, Editorial Tecnos, Madrid, 1981.

Facultad de Derecho y Ciencias Sociales - Universidad de Valparaíso - Chile 